\title{
Saúde mental de profissionais de enfermagem durante a pandemia de COVID-19: recursos de apoio
}

\author{
Mental health of nursing professionals during the COVID-19 pandemic: support resources
}

Salud mental de profesionales de enfermería durante la pandemia de COVID-19: recursos de apoyo

\author{
Aline Marcelino Ramos-Toescher ${ }^{1}$ (1) \\ Jamila Geri Tomaschewisk-Barlem ${ }^{2}$ (1) \\ Edison Luiz Devos Barlem² (D) \\ Janaína Sena Castanheira² (B) \\ Rodrigo Liscano Toescher $^{1}$ (D)
}

\section{Universidade Federal do Rio Grande. Rio} Grande, RS, Brasil.

2.Universidade Federal do Rio Grande, Escola de Enfermagem. Rio Grande, RS, Brasil.

\section{Autor correspondente:}

Aline Marcelino Ramos Toescher.

E-mail: alineramosenf@gmail.com

Recebido em 13/07/2020.

Aprovado em 14/08/2020.

DOl:https://doi.org/10.1590/2177-9465-EAN-2020-0276

\section{Resumo}

Objetivo: refletir sobre as implicações da pandemia de coronavírus na saúde mental dos profissionais de enfermagem e os principais recursos de apoio em desenvolvimento. Método: artigo reflexivo, produzido com base na formulação discursiva acerca da saúde mental dos profissionais de enfermagem frente a pandemia de coronavírus, correlacionado com as atuais recomendações de suporte psicológico do Ministério da Saúde, Organização Mundial da Saúde, Organização Pan-Americana da Saúde e Fundação Oswaldo Cruz. Resultados e discussão: em resposta à pandemia, uma crise em saúde mental pode estar a ocorrer entre os profissionais de enfermagem. Por estarem, diretamente, ligados ao atendimento de casos do novo coronavírus, experienciam situações estressoras, adicionais àquelas já vivenciadas nos serviços de saúde, incluindo preocupações, medo e insegurança com a saúde de si e da população. Como resultado, foi possivel refletir acerca das principais implicações da pandemia para os profissionais de enfermagem e os principais recursos de apoio em desenvolvimento, especialmente relacionados a identificação e manejo de situações estressantes. Conclusões e implicações para a prática: uma série de recursos de apoio úteis aos profissionais de enfermagem foram reunidos, com o objetivo de subsidiar estratégias para enfrentar as implicações da pandemia de coronavírus na saúde mental dos profissionais de enfermagem.

Palavras-chave: Pandemias; Coronavírus; Saúde Mental; Sistemas de Saúde; Enfermagem.

\section{Abstract}

Objective: to reflect upon the coronavirus pandemic's implications on nursing professionals' mental health and the main support resources in development. Method: reflection paper, based on a discussion about the mental health of nursing professionals in the context of the coronavirus pandemic, correlated with the current psychological support recommendations provided by the Brazilian Ministry of Health, World Health Organization, Pan American Health Organization, and Oswaldo Cruz Foundation. Results and discussion: a mental health crisis may be affecting nursing professionals in response to the pandemic. As these workers provide direct care to individuals infected by the novel coronavirus, in addition to the already stressful situations faced within healthcare services, they also experience concerns, fear, uncertainty about their health and that of the population. Hence, a reflection upon the pandemic's main implications for nursing professionals is presented here along with the main resources in development to support these workers, emphasizing the need to identify and adequately manage stressful situations. Conclusions and implications for practice: this study presents various resources to support nursing professionals coping with the coronavirus pandemic's mental health implications.

Keywords: Pandemics; Coronavirus; Mental Health; Health Systems; Nursing.

\section{Resumen}

Objetivo: reflexionar sobre las implicaciones de la pandemia de coronavirus en la salud mental de profesionales de enfermería y los principales recursos de apoyo en desarrollo. Método: artículo de reflexión, producido con base en la formulación discursiva sobre la salud mental de profesionales de enfermería ante la pandemia, correlacionado con las actuales recomendaciones de apoyo psicológico del Ministerio de la Salud, Organización Mundial de la Salud, Organización Panamericana de la Salud y Fundación Oswaldo Cruz. Resultados y discusión: en respuesta a la pandemia, puede estar ocurriendo una crisis de salud mental entre profesionales de enfermería, que experimentan situaciones estresantes, además de la rutina de los servicios de salud, incluidas las preocupaciones, el miedo y la inseguridad sobre la salud de ellos mismos y de la población. Fue posible reflexionar sobre las principales implicaciones de la pandemia para los profesionales de enfermería y los principales recursos de apoyo en desarrollo, especialmente relacionados con la identificación y el manejo de situaciones estresantes. Conclusiones e implicaciones para la práctica: recursos de apoyo útiles a los profesionales de enfermería fueron reunidos con el objetivo de apoyar estrategias para enfrentar las implicaciones de la pandemia en la salud mental de profesionales de enfermería.

Palabras clave: Pandemias; Coronavirus; Salud Mental; Sistemas de Salud; Enfermería. 


\section{INTRODUÇÃO}

Coronavírus é uma família de vírus responsáveis por quadros de infecções respiratórias que se apresentam como a segunda principal causa de resfriado comum (após rinovírus). Existem sete coronavírus humanos (HCoVs) conhecidos, entre eles o SARS-COV (que causa síndrome respiratória aguda grave), o MERS-COV (síndrome respiratória do Oriente Médio) e o SARS-CoV-2 (vírus que causa a doença coronavirus disease 2019 - COVID-19). O novo agente do coronavírus foi descoberto em 31 de dezembro de 2019, após casos registrados em Wuhan, na China. ${ }^{1,2}$

A Organização Mundial da Saúde (OMS) declarou, em 30 de janeiro de 2020, que o surto da doença causada pelo novo coronavírus se tornou uma emergência de Saúde Pública de importância Internacional. Em 11 de março de 2020, a COVID-19 foi caracterizada pela OMS como uma pandemia por conta da sua rápida expansão no mundo. ${ }^{2}$

No Brasil, ainda não há certeza quando ocorreram os primeiros casos. No entanto, o primeiro caso diagnosticado de COVID-19, oficialmente divulgado, aconteceu em São Paulo, em 25 de fevereiro de 2020, e a primeira morte relacionada ao vírus, foi relatada em 16 de março de 2020. Em 22 de maio de 2020 , o Brasil se tornou o segundo país no mundo com o maior número de casos, estando atrás somente dos Estados Unidos. ${ }^{1}$

Nesse sentido, a pandemia de COVID-19 é uma emergência global e já contaminou mais de 14 milhões de pessoas no mundo, totalizando mais de 600 mil óbitos. Assim, com o intuito de reduzir a propagação desse vírus, diversas mudanças no funcionamento da sociedade se tornaram emergentes, resultando em um bloqueio de mais da metade da humanidade. ${ }^{2}$

Essa doença representa um evento infeccioso de saúde pública em larga escala que modifica radicalmente as estruturas e investimentos dos sistemas de saúde no mundo, tornandose um acontecimento crítico que a comunidade internacional registra na história. Como características únicas desse evento, destacam-se a suspeita da sua origem na interface humanoambiente-animal e sua rápida expansão como resultado de níveis sem precedentes da interconectividade humana, mobilidade e comércio global. ${ }^{3,4}$

No Brasil, um levantamento recente publicado pela Associação de Medicina Intensiva Brasileira (AMIB) calcula que pelo menos $75 \%$ dos brasileiros contam apenas com o Sistema Único de Saúde (SUS), cuja disponibilidade de leitos de UTI (Unidade de internação intensiva) são de 22.844. No que concerne à rede privada, que atende em média $25 \%$ da população, o número de leitos de UTI é semelhante, totalizando 23.004. Tais números acabam por expor a desigualdade existente entre as redes públicas e privadas, e revelam uma fraqueza na saúde pública nacional. ${ }^{5}$

Com base nessa situação, um possível colapso na capacidade hospitalar e do sistema de saúde como um todo, torna-se palco de grande preocupação, principalmente quando somos levados a refletir sobre as limitações de leitos hospitalares, escassez de recursos humanos, materiais e dentre tantos outros elementos essenciais para o gerenciamento do surto. De maneira geral, o despreparo dos países para o enfrentamento da doença deu margem para a visibilidade de uma série de fragilidades relacionadas aos sistemas de saúde, que apesar de insistentemente existentes, pareciam "esquecidas".

Infelizmente, entre as fragilidades destacadas em um cenário nada equilibrado, outro aspecto tornou-se preocupante: a saúde mental dos profissionais de enfermagem. ${ }^{6}$ Embora a resposta a COVID-19 até o momento tenha se concentrado principalmente em conter a disseminação e prevenir a mortalidade, a pandemia mostrou também, que possui potencial para criar uma crise de sofrimento psicológico de grande repercussão no sistema de saúde mental. ${ }^{7}$

Segundo a OMS não existe uma definição "oficial" de saúde mental, uma vez que uma série de diferenças culturais, julgamentos subjetivos, e teorias relacionadas, acabam por afetar no modo como a "saúde mental" é definida. Portanto, a sua compreensão é mais ampla do que apenas a ausência de transtornos mentais, já que pode ser utilizada como um termo capaz de descrever o nível de qualidade de vida cognitiva ou emocional de um indivíduo, incluindo a sua capacidade de apreciar a vida e procurar um equilíbrio entre as atividades e os esforços para atingir a resiliência psicológica. ${ }^{8}$

Fazem parte desse contexto, uma gama de profissionais de saúde que atuam diretamente no atendimento de casos de COVID-19. Na linha de frente, estão os profissionais de enfermagem, cuja profissão emergiu como prática social associada aos elementos que compõem a vida humana nos seus múltiplos aspectos, com base na prevenção, promoção e reabilitação da saúde. ${ }^{6}$ Compreendem a maior categoria profissional da área, e ao permanecerem 24 horas ao lado dos pacientes, estão mais susceptíveis aos possíveis impactos psicológicos da pandemia. ${ }^{6}$

Esses profissionais, portanto, tornam-se facilmente alvos de vivencias estressoras no contexto de pandemias como: sobrecarga, fadiga, exposição a mortes em larga escala, frustações relacionadas a qualidade da assistência, ameaças, agressões e risco aumentado de serem infectados. Nesse caso, emergem o medo e a incerteza que podem influenciar de forma negativa no comportamento e bem-estar geral desses profissionais e, consequentemente, interferir na sustentação da qualidade dos cuidados em saúde destinados à população. ${ }^{7,9}$

Em resposta a atual crise, foram implementadas formas inovadoras de prestação de serviços de saúde mental e novas iniciativas de apoio psicossocial aos profissionais de saúde que, até então, conviviam com a falta de investimentos em promoção, prevenção e assistência à saúde mental.

Assim sendo, este estudo possui como relevância o seu teor reflexivo acerca dos efeitos da pandemia COVID-19 na saúde mental dos profissionais de enfermagem e sua abordagem referente ao o que está sendo realizado para a sua mitigação. Tem-se como objetivo: refletir sobre as implicações da pandemia 
de coronavírus na saúde mental dos profissionais de enfermagem e os principais recursos de apoio em desenvolvimento.

\section{MÉTODO}

Artigo de reflexão, produzido com base na formulação discursiva acerca da saúde mental dos profissionais de enfermagem frente a pandemia de coronavírus, correlacionado com as atuais recomendações de suporte psicológico do Ministério da Saúde, Organização Mundial da Saúde, Organização Pan-Americana da Saúde e Fundação Oswaldo Cruz.

Ainda, a partir do contexto e da relevância reflexiva sobre o tema, precisou-se obter um conjunto de informações atualizadas nos campos de saúde mental em enfermagem e surtos de infecções virais, através de periódicos disponibilizados nas bases de dados: Scientific Electronic Library Online (SCIELO), SAGE Journals e Medical Literature Analysis and Retrievel System Online (MEDLINE), utilizando-se os DeCS / MeSH: pandemics; coronavirus; mental health; nursing; e Disease Outbreaks. Sem limite de temporalidade.

\section{RESULTADOS E DISCUSSÃO}

Para lidar com os impactos na saúde mental da população e profissionais de enfermagem, uma série de informações sobre o gerenciamento de emoções, tem recebido destaque mediante a pandemia do novo coronavírus. Dessa maneira, autoridades sanitárias, especialistas em saúde mental e psiquiatria, organizações ligadas à saúde, e cientistas de diferentes localidades no mundo, estão desenvolvendo importantes orientações para realização de práticas alinhadas às demandas do atual contexto, que serão discutidas nessa seção.

\section{Implicações da pandemia COVID-19 na saúde mental dos profissionais de enfermagem}

Como visto, surtos de doenças infecciosas, como a COVID-19, podem sobrecarregar os sistemas de saúde e gerar uma série de sentimentos na população e profissionais de saúde, com destaque para os profissionais de enfermagem, como angústia, medo e incerteza. Em resposta ao surto infeccioso, os aspectos psicológicos, físicos e comportamentais podem receber influências negativas e ocasionar alguns sintomas adversos como insônia, insegurança, sentimento de incapacidade, tristeza, aumento do uso de álcool, tabaco e outras drogas, falta de energia e dores em geral. ${ }^{10,11}$

Ademais, os profissionais de saúde, e de modo especial, os profissionais de enfermagem enfrentam desafios adicionais durante surtos de doenças infecciosas, incluindo a sobrecarga de serviço, escassez de recursos humanos e materiais, incerteza da eficácia de tratamentos utilizados e ainda preocupações com o gerenciamento da própria saúde, e a de seus familiares e pacientes. ${ }^{6}$

Para agravar ainda mais a situação de angústia, precisam lidar constantemente com um arsenal de informações falsas provenientes das mídias sociais e tradicionais, que acabam por desviar e enfraquecer o comportamento da população em relação aos cuidados com a própria saúde.

Na literatura, aspectos traumáticos e estressantes já foram anteriormente reconhecidos entre enfermeiros e médicos envolvidos em surtos globais como SARS, MERS e Ebola, responsáveis pelo aumento de burnout, fadiga, menor satisfação no trabalho, sofrimento moral e elevados níveis de estresse. Portanto, torna-se claro que os prestadores de cuidados de saúde, como enfermeiros, podem desenvolver distúrbios psiquiátricos de curto e longo prazo importantes após vivenciarem eventos epidêmicos estressantes. ${ }^{12,13}$

Em 2003, durante o surto de SARS-CoV em Cingapura, 27\% dos profissionais de saúde relataram sintomas psiquiátricos. A equipe de enfermagem que prestou serviços relacionados ao MERS mostrou sintomas de transtorno de estresse pós-traumático após o surto coreano de 2015 , especialmente relacionado a sensação de isolamento interpessoal e o medo de contágio e transmissão entre familiares. De modo semelhante, durante os surtos de Ebola na Serra Leoa, em 2014, e na República Democrática do Congo em 2018, a equipe de enfermagem e demais equipes de saúde, que atuavam em contato direto com pacientes infectados, relataram altos níveis de ansiedade e estigma. ${ }^{13}$

Ainda, em setembro de 2009, 469 profissionais de saúde de um hospital-escola, na Grécia, completaram um questionário de 20 itens sobre preocupações e inquietações sobre a nova pandemia de gripe $\mathrm{A} / \mathrm{H} 1 \mathrm{~N} 1$. Como resultado, mais da metade desses profissionais, incluindo enfermeiros $(56,7 \%)$ relataram preocupação com a pandemia de influenza com um grau de ansiedade moderadamente alto. A preocupação mais frequente foi a infecção de familiares e amigos e as consequências da doença para a saúde $(54,9 \%){ }^{13}$

Como aspecto agravante para identificação de problemas em saúde mental, enfermeiros e médicos relataram ainda, que sentiam dificuldade em perceber possíveis problemas psicológicos em seus colegas de trabalho, especialmente pela dificuldade de interação e comunicação entre os membros da equipe, atribuídas ao uso contínuo de roupas de proteção individual e ao próprio distanciamento exigido. ${ }^{10}$

Já no decorrer da pandemia COVID-19, os profissionais de enfermagem vêm apresentando também, altos níveis de sofrimento psicológico, a saber: No Canadá, 47\% desses profissionais, relataram a necessidade de apoio psicológico; Na República Popular da China, os profissionais relataram altas taxas de depressão (50\%), ansiedade (45\%) e insônia (34\%); e, por fim, no Paquistão, um grande número desses profissionais relataram sofrimento psicológico moderado (42\%) a grave (26\%). ${ }^{14}$

Mediante as informações obtidas, cumpre destacar que, instrumentos psicométricos direcionados, especificamente aos profissionais de enfermagem, relacionados aos impactos psicológicos de COVID-19 ainda são inexistentes. Recentemente, foram validadas as escalas The Fear of COVID-19 Scale, ${ }^{15}$ para 
população Iraniana e a COVID Stress Scales, ${ }^{16}$ para a população norte-americana e canadense, cuja finalidade consiste em identificar pessoas que precisem de serviços de saúde mental relacionados a pandemia. Apesar de obterem boas propriedades psicométricas, nenhuma delas é direcionada para enfermeiros.

Contudo, diante de experiências já divulgadas num contexto mundial, percebe-se a urgência atrelada à antecipação dos sistemas de saúde, em identificarem e responderem às necessidades de saúde mental de pacientes, familiares e, principalmente dos profissionais de saúde. A ênfase está nos profissionais de enfermagem, que atuam na linha de frente no combate às doenças emergentes, ou seja, aqueles que atuam em contato direto com indivíduos infectados, com vistas no provimento de recursos de apoio adequados e efetivos para o manejo das situações. ${ }^{17,18}$

\section{Recursos de apoio em saúde mental na pandemia COVID-19}

Em decorrência da pandemia do novo coronavírus, intervenções psicológicas voltadas para os profissionais de enfermagem vêm desempenhando um papel extremamente importante para configuração do atual cenário. Assim, uma série de serviços psicológicos, realizados por meios de tecnologia da informação e comunicação, tem recebido destaque, principalmente por se constituírem de mecanismos relevantes para o acolhimento de queixas relacionados à saúde mental. ${ }^{7}$

As lições sobre os impactos da COVID-19 na saúde mental de profissionais de enfermagem atuantes em outros países, em conjunto com a percepção da própria realidade, tornaram evidente, a relevância da implementação de serviços estratégicos de atenção psicossocial, baseados em evidências, como forma de mitigar o estresse e o sofrimento intenso, além de prevenir futuros agravos. ${ }^{19}$

Nesse sentido, as ações de saúde mental precisam ser recomendadas desde a primeira fase de resposta ao surto. $O$ atendimento remoto aos profissionais, por exemplo, tem demonstrado inúmeras vantagens no oferecimento de suporte emocional, uma vez que corrobora com as recomendações de distanciamento social, quarentena e/ou isolamento domiciliar. ${ }^{19}$ Desse modo, é possível evitar a circulação desnecessária e, ao mesmo tempo, garantir atendimento psicológico com qualidade.

Nessa circunstância, o cenário da pandemia COVID-19 exige a reorganização das formas de atenção e cuidado, que precisam estar direcionados para as necessidades emergentes e prioridades de ações, que como consequência, colocam em evidência os serviços de Telemedicina e Telessaúde realizados para manejo clínico dos pacientes, suporte profissional e organização do Sistema Único de Saúde. É importante destacar a pré-existência de tais serviços, que se moviam de forma bastante lenta em direção à integração. ${ }^{20}$

Com base no suprimento de tais necessidades, existe forte mobilização de autoridades sanitárias, organizações ligadas à saúde, especialistas de universidades brasileiras e demais pesquisadores, para o fornecimento e facilidade de acesso dos profissionais de saúde, incluindo enfermeiros, as orientações, atendimentos e recomendações técnicas e científicas para o enfrentamento das situações desafiadoras atreladas ao novo contexto imposto pela pandemia. ${ }^{20-24}$

Em março de 2020, foi disponibilizado pelo Conselho Federal de Enfermagem (Cofen), um canal de atendimento ininterrupto, conduzido por enfermeiros especialistas em saúde mental, destinados a todos os profissionais de Enfermagem que necessitarem de ajuda emocional nesse período de crise. $\mathrm{O}$ atendimento é fornecido através de um chat on-line (disponível no site do Cofen e no hotsite Juntos Contra Coronavírus). ${ }^{22}$

Ainda, com foco na saúde mental dos milhares de profissionais de saúde diretamente ligados no combate ao COVID-19, o Ministério da Saúde investiu R $\$ 2,3$ milhões para oferta de um canal para teleconsulta psicológica, formada por profissionais de Psicologia e Psiquiatria entre os meses de maio e setembro de 2020. A iniciativa partiu do reconhecimento da necessidade de apoio a esses profissionais que, pela natureza do trabalho, lidam diariamente com condições adversas e, portanto, podem se sentir na condição de sofrimento psíquico. Havendo a necessidade de intervenção farmacológica, o profissional pode ser referenciado para atendimento presencial. ${ }^{23}$

Nessa perspectiva, é importante destacar as contribuições que o Ministério da Saúde vem desenvolvendo ao utilizar o potencial da Telemedicina e Telessaúde para a saúde da população brasileira, como o canal de teleconsulta para COVID-19 (TeleSUS) e o serviço de teleconsulta psicológica (TelePsi) para profissionais como médicos, enfermeiros, técnicos de enfermagem, fisioterapeutas, fonoaudiólogos, nutricionistas, biomédicos e farmacêuticos envolvidos nos atendimentos de coronavírus.

Outros serviços de suporte psicológico destinados aos profissionais de enfermagem englobam também, ${ }^{24}$ ações psicoeducativas através do fornecimento de cartilhas virtuais, plataformas com guias informativos, softwares, vídeos, áudios, vídeo aulas, manuais, e-books, contas de Instagram e WhatsApp disponibilizados por conselhos e grupos de psicólogos voluntários, além do fornecimento de plantões psicológicos realizados em hospitais universitários de várias localidades do Brasil.

No Quadro 1, foram sistematizadas as principais situações que podem impactar negativamente na saúde mental dos profissionais de enfermagem relacionadas ao enfrentamento da COVID-19, além das recomendações desenvolvidas pela Organização Mundial da Saúde (OMS), governos e entidades profissionais acerca de como prevenir/mitigar as consequências psicológicas da pandemia.

\section{CONSIDERAÇÕES FINAIS E IMPLICAÇÕES PARA A PRÁTICA}

Com base nos acontecimentos históricos de surtos infecciosos já registrados na história, é possível identificar que uma proporção 
Quadro 1. Principais implicações da pandemia COVID-19 na saúde mental dos profissionais de enfermagem e recursos de apoio difundidos, 2020.

\begin{tabular}{|c|}
\hline Implicações da pandemia COVID-19 para profissionais de enfermagem \\
\hline Principais fatores \\
\hline - Alta demanda de atendimento; ${ }^{15}$ \\
\hline - Exposição prolongada em ambientes críticos; ${ }^{15,21}$ \\
\hline - Risco contínuo de infecção; ${ }^{15}$ \\
\hline - Equipamento de proteção individual (desconforto, limitações na mobilidade e comunicação, efetividade e escassez); ${ }^{15,21}$ \\
\hline - Gerência do sofrimento de pacientes e familiares; ${ }^{15}$ \\
\hline - Falta de contato com a família; ${ }^{15}$ \\
\hline - Conflitos interpessoais; ${ }^{15}$ \\
\hline - Pensamentos recorrentes sobre a epidemia; e relacionados a morte e ao morrer; ${ }^{15}$ \\
\hline - Estigma por trabalhar com pacientes com COVID-19; ${ }^{15,21}$ \\
\hline - Desmistificação de notícias falsas constantemente. ${ }^{15,21}$ \\
\hline Reações emocionais \\
\hline $\begin{array}{l}\text { - Medo, pesar, frustração, culpa, raiva, exaustão, incerteza, desesperança, sofrimento moral, burnout, estresse, ansiedade, } \\
\text { sintomas depressivos, negação, impotência, sentimento de vulnerabilidade e irritabilidade, }{ }^{17,20}\end{array}$ \\
\hline - Alterações ou distúrbios de apetite (falta de apetite ou apetite em excesso); ${ }^{15,20}$ \\
\hline - Alterações ou distúrbios do sono (insônia, dificuldade para dormir ou sono em excesso, pesadelos recorrentes); ${ }^{12,20}$ \\
\hline - Uso de drogas lícitas e ilícitas. ${ }^{20}$ \\
\hline Estratégias de enfrentamento/recursos de apoio \\
\hline $\begin{array}{l}\checkmark \text { Atenda às necessidades básicas: alimente-se, hidrate-se e durma regularmente para otimizar sua capacidade de cuidar de } \\
\text { si e dos outros; }{ }^{15,24}\end{array}$ \\
\hline $\begin{array}{l}\sqrt{ } \text { Evite hábitos prejudiciais - Cuidado com o uso abusivo de tabaco, bebidas alcoólicas e outras drogas como forma de fugir } \\
\text { da angústia;12,24 }\end{array}$ \\
\hline $\begin{array}{l}\checkmark \text { Respeite seu tempo: faça pausas, permita-se fazer algo não relacionado ao trabalho que considere reconfortante, } \\
\text { divertido ou relaxante. Atividades de descanso, exercícios físicos e relaxamento são importantes aliados contra o estresse e } \\
\text { ansiedade; }{ }^{12,24}\end{array}$ \\
\hline $\begin{array}{l}\checkmark \text { Crie uma rotina de trabalho e autocuidado: procure realizar atividades prazerosas e significativas, isso ajudará o dia a } \\
\text { acontecer de um jeito mais organizado e tranquilo; }{ }^{: 15,24}\end{array}$ \\
\hline $\begin{array}{l}\checkmark \text { Permaneça conectado com sua rede sócio afetiva: conecte-se com familiares, colegas e amigos (eles são uma âncora de } \\
\text { apoio fora do sistema de saúde), dê e receba apoio, reduza o sentimento de isolamento;; } 15,24\end{array}$ \\
\hline $\begin{array}{l}\checkmark \text { Mantenha-se atualizado: confie em fontes confiáveis de informação, cuidado com o excesso. Participe de reuniões de } \\
\text { trabalho onde informações relevantes são compartilhadas; }{ }^{15,24}\end{array}$ \\
\hline $\begin{array}{l}\checkmark \text { Auto check-ins: monitore-se ao longo do tempo em busca de sintomas de depressão ou transtorno de estresse: tristeza } \\
\text { prolongada, dificuldade para dormir, lembranças intrusivas, desesperança. Converse com um colega, supervisor ou procure } \\
\text { ajuda profissional, se necessário; } 15,24\end{array}$ \\
\hline $\begin{array}{l}\checkmark \text { Pratique a Resiliência - Reflita sobre as dificuldades enfrentadas e o que pode aprender com elas, ressignifique sua } \\
\text { experiência. Retome estratégias de enfretamento já utilizadas em crises anteriores; }{ }^{12}\end{array}$ \\
\hline$\checkmark$ Mantenha sua fé e atividades religiosas e/ou espirituais, caso façam parte de sua rotina; ${ }^{15,24}$ \\
\hline $\begin{array}{l}\checkmark \text { Busque um profissional de saúde quando as estratégias utilizadas não estiverem sendo suficientes para sua estabilização } \\
\text { emocional: Informe-se quanto a disponibilidade de serviços em saúde mental destinados a você. }{ }^{15,24}\end{array}$ \\
\hline
\end{tabular}

Fonte: Dados da pesquisa, 2020. 
significativa de profissionais de enfermagem experimentou ansiedade moderadamente alta no desenvolvimento das suas atividades. Esse fato é bastante semelhante ao que estamos vivenciando na atualidade, e que nos dá margem para reflexão sobre as condições de preparo psicológico desses profissionais em emergências de larga escala, como é o caso da COVID-19.

Compreende-se também, que o surto que estamos vivenciando é multidimensional, com impactos em todas as dimensões funcionais, incluindo físico, emocional, econômico, social e psicológico. Sendo assim, embora o medo e a ansiedade sejam esperados durante esse período, também é importante entendermos e identificarmos de maneira precoce seus extremos, como as formas de medo e ansiedade exacerbadas.

Cumpre destacar ainda, que a presença de estresse, ansiedade e outros sintomas psicológicos possuem um significado ainda mais delicado no contexto de atuação de profissionais de enfermagem, pois estes encontram-se em uma posição favorável para o acolhimento, escuta atenciosa e conforto dos pacientes que necessitam de assistência. Todavia, ao estarem emocionalmente abalados, podem tornar a natureza do cuidado enfraquecida.

Por outro lado, o emprego de medidas globais de saúde mental colocou em evidência, alguns recursos de apoio importantes para o manejo de situações estressantes, especialmente pela possibilidade de melhor preparar os profissionais de enfermagem para o desenvolvimento efetivo de suas atividades em condições de extrema vulnerabilidade e angústia, como nos casos de surtos epidemiológicos atuais e futuros.

Ainda, vimos pela primeira vez, o uso da psicoterapia no teleatendimento em um contexto de pandemia, demonstrando um avanço significativo na área de saúde mental que, por sua vez, abre portas para uma série de atividades futuras voltadas para os serviços de Telemedicina e Telessaúde.

Concluímos que, para melhor entendimento das repercussões psicológicas e psiquiátricas de uma pandemia, é preciso levar em consideração as principais implicações e emoções envolvidas antes, durante e após o evento. Assim, mais pesquisas sobre os impactos da pandemia COVID-19 na saúde mental dos profissionais de enfermagem precisam ser realizadas, uma vez que a utilização do conhecimento e experiência prévia de situações semelhantes auxiliam no direcionamento de ações e recursos efetivos.

Como fator limitante para análise de aspectos teóricos, destaca-se a escassez de estudos científicos de campo que abordem de modo direcionado, os impactos da pandemia do novo coronavírus à saúde mental dos profissionais de enfermagem. Haja vista que grande parte dos estudos são realizados de modo a envolver todos os profissionais de saúde, sem considerar as particularidades de cada profissão.

\section{CONTRIBUIÇÕES DOS AUTORES}

Desenho do estudo de reflexão: Aline Marcelino RamosToescher.

Análise e Interpretação reflexiva: Aline Marcelino RamosToescher. Jamila Geri Tomaschewisk-Barlem. Edison Luiz Devos Barlem.

Redação e revisão crítica do manuscrito: Aline Marcelino Ramos-Toescher. Jamila Geri Tomaschewisk-Barlem. Edison Luiz Devos Barlem. Janaína Sena Castanheira. Rodrigo Liscano Toescher.

Aprovação da versão final: Aline Marcelino Ramos-Toescher. Jamila Geri Tomaschewisk-Barlem. Edison Luiz Devos Barlem. Janaína Sena Castanheira. Rodrigo Liscano Toescher.

Responsabilidade pelo conteúdo intelectual, acurácia e integridade de quaisquer partes do artigo: Aline Marcelino RamosToescher. Jamila Geri Tomaschewisk-Barlem. Edison Luiz Devos Barlem. Janaína Sena Castanheira. Rodrigo Liscano Toescher.

\section{EDITOR ASSOCIADO}

\author{
Cristina Rosa Baixinho
}

\section{REFERÊNCIAS}

1. Ministério da Saúde (BR). O que é coronavírus? [Internet]. Brasília: Ministério da Saúde; 2020 [citado 2020 Maio 5]. Disponível em: https://www.saude.gov.br/o-ministro/746-saude-de-a-a-z/46490-novocoronavirus-o-que-e-causas-sintomas-tratamento-e-prevencao-3

2. World Health Organization. Novel coronavirus (COVID-19) [Internet]. Geneva:WHO;2020 [citado 2020 Mar 26]. Disponível em: https://www. who.int/emergencies/diseases/novel-coronavirus-2019

3. Carver PE, Phillips J. Novel Coronavirus (COVID-19): what you need to know. Workplace Health Saf. 2020;68(5):250. http://dx.doi. org/10.1177/2165079920914947. PMid:32299328.

4. Amuasi JH, Walzer C, Heymann D, Carabin H, Huong LT, Haines A et al. Calling for a COVID-19 one health research coalition. Lancet. 2020;395(10236):1543-4. http://dx.doi.org/10.1016/S0140-6736(20)31028-X. PMid:32386563.

5. Associação de Medicina Intensiva Brasileira. Atualizações sobre o Coronavírus COVID-19 [Internet]. São Paulo: AMIB;2020 [citado 2020 Mar 26]. Disponível em: https://www.amib.org.br/fileadmin/user_upload/ amib/2020/abril/28/dados_uti_amib.pdf

6. Souza LPS, Souza AG. Enfermagem brasileira na linha de frente contra o novo Coronavírus: quem cuidará de quem cuida? J Nutr Health [Internet]. 2020; [citado 2020 Jun 28];10:e20104005. Disponível em: https://periodicos.ufpel.edu.br/ojs2/index.php/enfermagem/article/ view/18444/11237

7. Schmidt B, Crepaldi MA, Bolze SDA, Neiva-Silva L, Demenech LM Saúde mental e intervenções psicológicas diante da pandemia do novo coronavírus (COVID-19). Estud Psicol. 2020;37:e200063. http:// dx.doi.org/10.1590/1982-0275202037e200063.

8. Gaino LV, Souza J, Cirineu CT, Tulimosky TD. O conceito de saúde mental para profissionais de saúde: um estudo transversal e qualitativo. SMAD Rev Eletrônica Saúde Mental Álcool Drog. 2018;2:108-16. http:// dx.doi.org/10.11606/issn.1806-6976.smad.2018.149449.

9. Ornell F, Schuch JB, Sordi AO, Kessler FHP. "Pandemic fear" and COVID-19: mental health burden and strategies. $\mathrm{Br} J$ Psychiatry. 2020;42(3):232-5. http://dx.doi.org/10.1590/1516-4446-2020-0008. PMid:32267343.

10. Torales J, O'Higgins M, Castaldelli-Maia JM, Ventriglio A. The outbreak of COVID-19 coronavirus and its impact on global mental health. Int J Soc 
Psychiatry.2020;66(4):317. http://dx.doi.org/10.1177/0020764020915212. PMid:32233719.

11. Pan American Health Organization. Mental health and psychosocial considerations during COVID-19 outbreak [Internet]. Geneva: PAHO; 2020 [citado 2020 Jun 28]. Disponível em: https://www.paho.org/en/ documentos/consideraciones-psicosociales-salud-mental-durantebrote-covid-19

12. Smith MW, Smith PW, Kratochvil CJ, Schwedhelm S. The psychosocial challenges of caring for patients with Ebola virus disease. Health Secur. 2017; 15(1):104-9. http://dx.doi.org/10.1089/hs.2016.0068. PMid:28192056.

13. Goulia P, Mantas C, Dimitroula D, Mantis D, Hyphantis T. General hospital staff worries, perceived sufficiency of information and associated psychological distress during the $\mathrm{A} / \mathrm{H} 1 \mathrm{~N} 1$ influenza pandemic. BMC Infect Dis. 2010;10(1):322. http://dx.doi.org/10.1186/1471-2334-10-322. PMid:21062471.

14. United Nations. Policy brief: Covid-19 and the need for action on mental health [Internet]. 2020 [citado 2020 Jun 28]. Disponível em: https:// www.un.org/sites/un2.un.org/files/un_policy_brief-covid_and_mental_ health_final.pdf

15. Ahorsu DK, Lin CY, Imani V, Saffari M, Griffiths MD, Pakpour AH. The fear of COVID19 scale: development and initial validation. Int J Ment Health Addict. 2020;27:1-9. http://dx.doi.org/10.1007/s11469-02000270-8. PMid:32226353.

16. Taylor S, Landry C, Paluszek M, Fergus TA, Mckay D, Asmundson GJG. Development and Initial Validation of the COVID Stress Scales. J Anxiety Disord.2020;72:102232. http://dx.doi.org/10.1016/j.janxdis.2020.102232. PMid:32408047.

17. Choi KR, Heilemann MV, Fauer A, Mead M. A second pandemic: mental health spillover from the novel Coronavirus (COVID-19). J Am Psychiatr Nurses Assoc. 2020;26(4):340-3. http://dx.doi. org/10.1177/1078390320919803. PMid:32340586.

18. American Psychiatric Association. APA Offers Resources to Cope with COVID-19 [Internet]. Washington: APA; 2020 [citado 2020 Jun 28].
Disponível em: https://www.psychiatry.org/newsroom/news-releases/ apa-offers-resources-to-cope-with-covid-19

19. Duan L, Zhu G. Psychological interventions for people affected by the COVID-19 epidemic. Lancet Psychiatry. 2020;7(4):300-2. http://dx.doi. org/10.1016/S2215-0366(20)30073-0. PMid:32085840.

20. Sullivan AB, Kane A, Roth AJ, Davis BE, Drerup ML, Heinberg LJ. The COVID-19 crisis: a mental health perspective and response using telemedicine. J Patient Exp. 2020;7(3):295-301. http://dx.doi. org/10.1177/2374373520922747. PMid:32821785.

21. Jung SJ, Jun JY. Mental health and psychological intervention amid COVID-19 Outbreak: perspectives from South Korea. Yonsei Med J. 2020;61(4):271-2. http://dx.doi.org/10.3349/ymj.2020.61.4.271. PMid:32233168.

22. Resolução COFEN no 634, de 26 de março de 2020 (BR). Autoriza e normatiza a teleconsulta de enfermagem como forma de combate à pandemia provocada pelo novo coronavírus (Sars-Cov-2). Diário Oficial da União [periódico na internet], Brasília (DF), 27 mar 2020 [citado 2020 Jun 28]. Disponível em: http://www.cofen.gov.br/resolucao-cofenno-0634-2020_78344.html

23. Ministério da Saúde (BR). Profissionais do SUS já podem contar com suporte psicológico [Internet]. Brasília; 2020 [citado 2020 Jun 28]. Disponível em: https://www.saude.gov.br/noticias/agencia-saude/46906profissionais-do-sus-ja-podem-contar-com-suporte-psicologico

24. Fundação Oswaldo Cruz. Saúde mental e atenção psicossocial na pandemia COVID-19: recomendações para gestores [Internet]. Brasília: FIOCRUZ; 2020. Disponível em: https://www.fiocruzbrasilia.fiocruz.br/wp-content/ uploads $/ 2020 / 04 /$ Sa\%c3\%bade-Mental-e-Aten \%c3\%a7\%c3\%a3oPsicossocial-na-Pandemia-Covid-19-recomenda\%c3\%a7\%c3\%b5esgerais.pdf 